Ann. Zootech., I975, $24(3), 447-464$.

\title{
ÉTUDE DU BESOIN EN LYSINE DE LA TRUIE GESTANTE NULLIPARE
}

\author{
P. H. DUÉE et A. RÉRAT* \\ avec la collaboration technique de J. JUnc, M. Sirreat et J. I.tibost \\ Station de Recherches sur l'Elevage des Porcs, \\ * I.aboratoive de Physiologie de la Nutrition. \\ Centre national de Recherches zootcchniques, I. N. R. A., \\ 78350 Jouy in Josas
}

\section{RÉSUMÉ́}

Une étude a été entreprise dans le but de déterminer le besoin en lysine de la Truie gestante nullipare. A cet effet, 4 lots de 6 a 7 truies Largr-White dont un lot témoin de truies vicles ont été comparés pendant un cycle de reproduction sur la base des variations de poids des animaux, des performances de reproduction en relation avec la digestibilité et la rétention d'azote en fin de gestation (80 jours) et l'évolution des concentrations en acides aminés libres du sang. Le régime de base en gestation, contenant $3360 \mathrm{kcal}$ d'énergie digestible par kg de matière sèche, Io,5 p. Ioo le matières azotées et $0,23 \mathrm{p}$. roo de lysine et distribué à un niveau fixé à 2 ky par jour, est supplémenté par divers taux de L-lysine industrielle $(0,0,2,0,4$ p. 100 du régime) correspondant aux trois lots de truies gravides (lots $2,3,4)$. I.es truies vides diı lot I reçoivent le même régine que celles du lot 3. Pendant lá lactation, tous les animaux consomment, à volonté, un aliment ì 16 p. 100 de protéines.

L'addition de lysine au régime de kase pendant la gestation a pour effet d'accroitre le gain cle poids des truies pendant cette période (xain de gestation (kg) : lot $2: 39,7 ; \operatorname{lot} 3: 44,8 ; \operatorname{lot} 4$ : $49,8)$. Tar rapport au lot carencé en lysine, une supplémentation de 0,2 p. 100 cn cet acide amine permet d'augmenter le poids moyen des porcelets à la naissance (lot $2: 0,98 \mathrm{~kg} ; \operatorname{lot} 3: 1,25 \mathrm{~kg}$ ) sans pour autant er affecter le nombre (lot $2: 8,4 ;$ lot $3: 8,5$.$) . Mais la croissance réelle des truies$ reste inféricure à celle des truies vicles (gain net de gestation (kg) lot I :30,7; lot $2: 25,6 ; \operatorname{lot} 3$ : $28,2)$.

Un supplément plus important de lysine $(o,+p$. roo du régime) permet une croissance maternelle conforme it celle des truies vicles (gain net du lot $t: 33,7 \mathrm{~kg}$ ), sans amélioration des performances de reproduction à la mise bas (ncmbre de porcelets : 7,5 : poids moyen des porcelets : $1,2+\mathrm{kg}$ ). Ceci se traduit par une rétention azotée plus élevée en fin de gestation ( $6,2 \mathrm{~g}$ d'azote par jour dans le lot 4 contre $8,9,9,69,4$ respectivement dans les lots $1,2,3)$.

L'effet néfaste d'une carence en lysine pendant la gestation se répercute, de la mème manière, sur le poids des porcelets au moment du sevrage (nombre et poids moyen (kg) : lot $2: 7,5,5,73$; lot $3: 7,5,7,25 ; \operatorname{lot}+: 7,3,8,10)$.

L'étude des variations cles concentrations en acides aminés libres du sang souligne que le besoin en lysine est plus élevé en fin de gestation.

Enfin, les performances de croissance des porcelets, après leur serrage, ne sont pas affectées par la tencur en lysine du régine maternel de gestation mais il subsiste un retard, dans le lot carencé, au stade d'abattage à un poids fixe ( I $00 \mathrm{~kg}$ ).

L'inopportunité d'une diminution trop sévère de la teneur en lysine daus le régime maternel de gestation ressort des résultats de cette étude. 


\section{INTRODUCTION}

L'objectif principal, en matière de reproduction porcine, est de faire produire, aux truies, le maximum de porcelets à un poids leur permettant une croissance ultérieure normale. De ce fait, l'alimentation des mères durant le cycle de reproduction a fait l'objet d'importants travaux depuis une quinzaine d'années. A la lumière des calculs ou des expériences effectués par MOUSTGAARD (I959) et par SALMON-LEGAGNEUR (I965), montrant la faible quantité de matières azotées déposées in utero au cotrs de la gestation ou en constatant, avec POND et al., (Ig68 a et b, I969) et de GEETER et al., (r972), l'absence d'influence sur les résultats de reproduction d'une carence partielle ou totale de protéines, on peut penser que le besoin quantitatif et qualitatif en matières azotées de la Truie gestante est faible.

Cependant, on peut se demander si l'utilisation, pendant la gestation de sources azotées de faible valeur biologique ou même de régimes uniquement à base de céréales n'est pas susceptible de faire naître des carences en certains acides aminés indispensables. La connaissance précise des quantités d'acides aminés à fournir, quotidienne ment, à l'animal au cours de cette période est donc essentielle. Pourtant, peu d'expériences ont été réalisées sur le besoin en acides aminés de la Truie gestante et on peut considérer que les recommandations formulées par RIPPE. (rg67) restent encore les seules estimations valables. Or, ces normes, établies en fin de gestation, en se basant sur la rétention maximale d'azote corporel, peuvent avoir été surestimées, d'autant plus que, parallèlement à l'accroissement đe la rétention azotée, l'auteur ne note pas d'amélioration des performances de reproduction (nombre et poids des porcelets à la mise bas).

Dans cette optique, une étude a été réalisée sur le besoin en lysine de la Truie gestante nullipare en tenant compte des critères de reproduction, de la rétention azotée en fin de gestation en relation avec les variations des concentrations en acides aminés libres du sang à divers stades de la gestation.

\section{MATÉRIEL, ET MÉTHODES}

$$
\text { I. - Animaux }
$$

L'expérience a été entreprise sur 38 truies Large-White, d'âge ct do poids moyens à la puberté, respectivement de $25 \mathrm{I}$ jours et de $108 \mathrm{~kg}$. Les animaux ont été répartis en quatre lots dont un lot témoin de 6 truies vides (lot I), poursuivant leur croissance pendant le temps d'une gestation. Les truies des trois autres lots, saillies au premier cestrus, ont été soumises dès le lendemain de la saillie à des régimes ne différant que par le taux d’introduction de lysine supplémentaire. Sur l'ensemble des truies saillies, près de 40 p. Ioo restent infécondées ou avortent en cours de gestation et sont éliminées de l'expérience. Finalement, le nombre de truies ayant parachevé leur gestation est de 7 (lot 2) ou de 6 (lot 3 et lot 4 ).

$$
\text { 2. - Régimes }
$$

Les régimes expérimentaux de gestation sont isoazotés ( I 0,5 à I r p. Ioo de matières azotées totales) et isoénergétiques ( $360 \mathrm{kcal}$ d'énergie digestible par $\mathrm{kg}$ de matière sèche). Le niveau alimentaire est fixé à $2 \mathrm{~kg}$ d'aliment par jour. La source azotéc est constituéc d'une part, de 
tourteau de sésame dont on sait qu'il est carencé en lysine, et d'autre part, de monoglutamate de sodium, apportant un complément fixe d'azote non essentiel. Le taux de L-lysine supplémentaire, sous forme $\mathrm{HCl}$ est $0,0,25$ ou $0,50 \mathrm{p}$. 100 du régime respectivement pour les lots 2,3 et 4 . Le lot I (truies non gestantes) et le lot 3 reçoivent le même régime. La composition des régimes et les résultats d'analyse sont consignés dans le tableau $I$. lin lactation, par contre, tous les animaux reçoivent à volonté le même régime ( 6 p. I oo de matières azotées totales), à base d'orge, de tourteau de soja 45 , de farine de poisson et cle farine de luzerne et dont la composition et les caractéristiques sont rapportées dans le tableau i bis.

\section{TABIEAU I}

Composition et caractéristiques des régimes cxpérimentaux de gestation

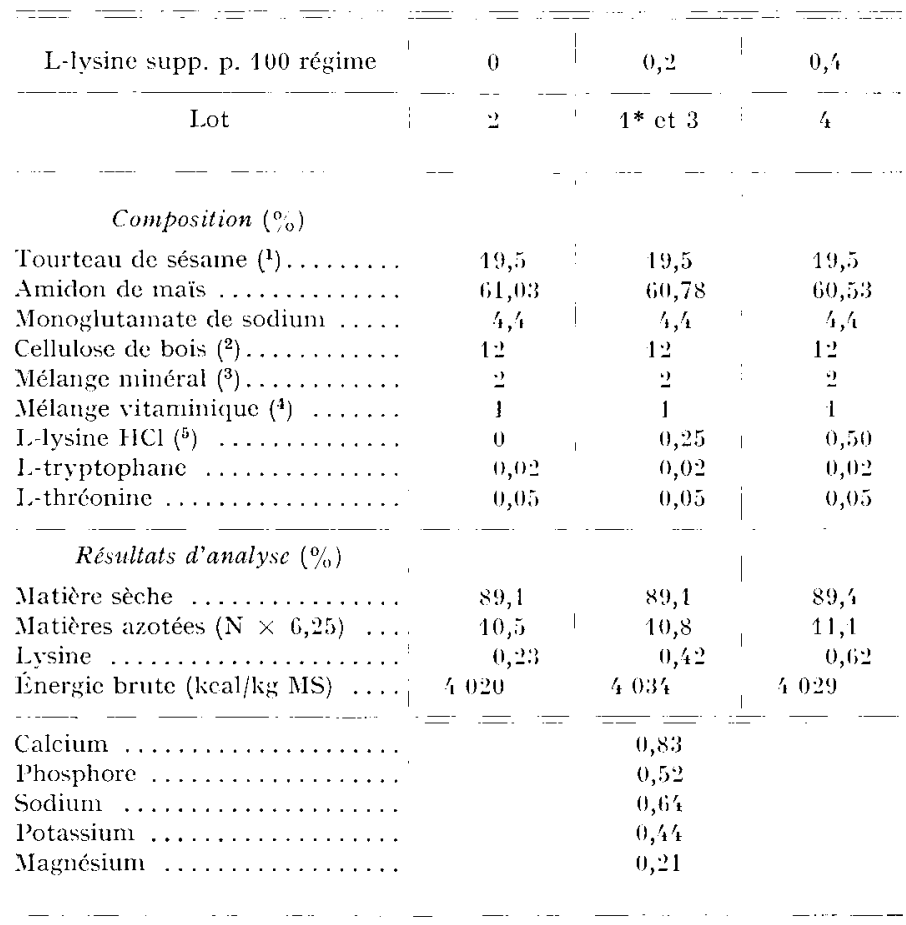

* : Truies non gestantes.

(1) Tourteau à 93,5 p. 100 de matière sèche, renfermant 12,9 p. 100 (le matières azotées. Teneur en lysine : $2,81 \mathrm{~g} / 16 \mathrm{~g} \mathrm{~N}$ (données non publićes).

(2) Cellulose purifiée extraite du bois.

(3) Mélange minćral (p. 100 de la ration) : phosphate bicalcique : 1,4 ; chlorure de potassium : 0,3 ; sulfate de magnésium : 0,2; oligocléments : 0,1 .

(4) Vélange vitaminique (p. 100 du mélange) : Vitamine $\mathrm{D}_{3}(100000)$ : 11,25 ; Riboflavine : 0,10 ; Acide ascorbique : 0,10 ; Nicotinamide : 0,20 ; panthothénate de calcium : 0,25 ; Vitamine A $(50000): 2,0$; Vitamine $\mathrm{B}_{12}$ (100 mg/kg) : 2,0; choline : 10,0 ; Glucose anhydre gsp. base.

(5) Produit commercial : 95 p. 100 de L-lysine $\mathrm{HCl}$ à 80 p. 100 de lysine 
TABLEAU I bis

Composition ot caractéristiques du végime de lactation (1)

Composition (")

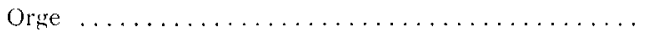

Tourteau de soja $\mathbf{t} \ldots \ldots \ldots \ldots \ldots \ldots \ldots \ldots \ldots \ldots$

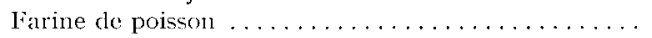

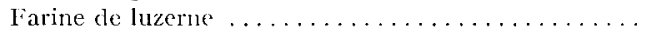

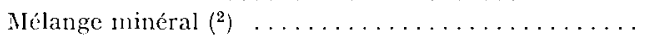

Mélange vitamini $\left({ }_{1}\right.$ ue $\left({ }^{3}\right) \quad \ldots \ldots \ldots \ldots \ldots \ldots \ldots$

Résultats d'analyse $(\%)$

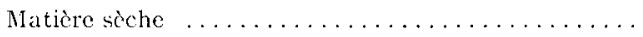

Matières azotécs $(\Lambda \times(i, 25) \ldots \ldots \ldots \ldots \ldots \ldots \ldots$

Jinergie brute $\ldots \ldots \ldots \ldots \ldots \ldots \ldots \ldots \ldots \ldots \ldots$

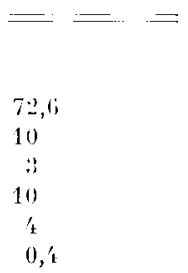

88,5

16,1

$4: 40 \mathrm{kcal} / \mathrm{kg}$.IS

(1) Régime commun à tous les animaux.

(2) Composition (on p. 100 du mélange) : phosphate bicalcique : 40 ; carbonate de calcium : 2.2 ; chlorure de sodium : 25 ; carbonate de magnćsium . 10 ; sulfate ferreux : 1 ; sulfate de zine: 1 ; sulfate cle manganèse : 0,1 ; sulfate de cuive : 0,05 ; sulfate de cobalt : 0,05 ; iodure de potassium stabilisé : 0,01 .

(3) Voir tableau 1.

\section{3. - Mesures effectuées}

Les variations de poids cles mères au cours de l'ensemble du cycle sont enregistrées ainsi que leurs performances de reproduction it la mise bas et au sevrage (35 jours).

Vers $1 \mathrm{e} 80^{\mathrm{e}}$ jour de gestation, 5 animaux de chaque lot sont soumis, après une semaine d'adaptation, à une période de 5 jours en cages de digestibilité pendant laquelle on récolte séparément les fèces et l'urine, dans le but de déterminer la digestibilité apparente des matières azotées ingérées et la rétention d'azote à ce stade physiologique.

En relation avec ce dernier critère, des prélèvements de sang ont été effectués au nivcau de lá vcine cáve, $6 \mathrm{~h} 30$ après le premier repas, à 3 stades de la gestation : à la saillie, sur 5 animaux, alors que les régimes expérimentaux n'ont pas cncore été distribués, vers 6o jours de gestation. sur 3 animaux par lot et vers go jours de gestation, sur 5 animaux par lot. En prélèvement de colostrum a, d'autre part, été effectué dans les 12 heures après le part. I-es concentrations en acides aminés libres du sang ont été déterminées, sur chaque échantilon, par chromatographie sur colonne, après épuisements successifs par l'éthanol à $82 \mathrm{p}$. roo, techniquue décrite par P'AWLAK et́ PION (1968).

\section{4. - Séparation et dosage dos acides aminés}

La séparation et le dosage des acides aminés ont été effectués sur un appareil automatique JLC-5 $\Lambda \mathrm{H}$ (Jeolco SA), d'après la méthode mise au point par Sirackmar, MoORE et STEIN (I958).

La séparation des acides aminés acides et neutres est réalisée en 6 heures au moyen de tampons citrates de lithium (BENsox et al., 1967), sur une colonne de $50 \times 0,8 \mathrm{~cm}$ de résine $\mathrm{I}_{\mathrm{C}} \mathrm{R}_{2}$ (fournie par Jeolco), maintenue d'abord a la températurc cle $33^{\circ} \mathrm{C}$ puis, 3 h $3^{\circ}$ plus tard, ì la température de $50^{\circ} \mathrm{C}$.

La concentration en lithium et le $\mathrm{pH}$ des tampons sont succossivement de $0,27 \mathrm{~N}, 2,80$; $0,18 \mathrm{~N}, 2,98 ; 0,3 \mathrm{~N}, 3,00 ; 0,3 \mathrm{~N}+18 ;$ le débit utilisé étant de $50 \mathrm{ml} / \mathrm{heure}$.

Les acides aminés basiques sont séparés en moins de 5 heures sur une colonne de $30 \times 0,8 \mathrm{~cm}$ de résine $\mathrm{ICR}_{2}$, à la température de $33^{\circ} \mathrm{C}$, pendant $2 \mathrm{~h} 44^{\circ}$, puis de $50^{\circ} \mathrm{C}$.

Les tampons utilisés sont successivement un tampon citrate de sodium o, $38 \mathrm{~N}, \mathrm{pH}+, 30$ à la température de $33^{\circ} \mathrm{C}$, puis un tampon citrate de sodium $0,35^{\mathrm{N}}, \mathrm{pH}_{5,28}$, à $50^{\circ} \mathrm{C}$. Le débit des tampons est de $73 \mathrm{ml}$ par heure. 
Dans tous les cas, la température du bain de réaction est de $95^{\circ} \mathrm{C}$, le débit de ninhydrine étant de $\mathbf{2} 2,6 \mathrm{ml} /$ heure, le débit de l'éluat double de celui de la ninhydrine.

La composition en acides aminés des différents aliments et du colostrum a été également déterminée par chromatographie sur colonne échangeuse d'ions, après hydrolyse acide (tabl. 2). Les acides aminés soufrés ont été dosés après oxydation performique des protéines. Les caractéristiques du dosage ont été, antérieurement, rapportées par Pion et FauconneaU (I966).

TABLEAU 2

Composition en acides aminés du régime de base de gestation (lot 2) et du végime de lactation

\begin{tabular}{|c|c|c|c|c|}
\hline & \multicolumn{2}{|c|}{ Aliment : gestation } & \multicolumn{2}{|c|}{ Lactation } \\
\hline & $\left|\begin{array}{c}\text { en } g \\
\text { pour } 16 \mathrm{gN}\end{array}\right|$ & $\begin{array}{l}\text { en p. } 100 \\
\text { du régime }\end{array}$ & $\begin{array}{c}\text { en } \mathrm{g} \\
\text { pour } 16 \mathrm{gN}\end{array}$ & $\begin{array}{l}\text { en p. } 100 \\
\text { du régime }\end{array}$ \\
\hline$\ldots \ldots \ldots \ldots \ldots$ & 2,37 & 0,23 & 4,63 & 0,80 \\
\hline Histidine $\ldots \ldots \ldots \ldots$ & 2,01 & 0,20 & 2,13 & 0,37 \\
\hline Arginine $\ldots \ldots \ldots \ldots \ldots$ & 10,03 & 0,97 & 5,55 & 0,96 \\
\hline Acide aspartique $\ldots \ldots \ldots$ & 7,06 & 0,69 & 7,82 & 1,35 \\
\hline Thréonine $\ldots \ldots \ldots \ldots$ & 3,58 & 0,35 & 3,83 & 0,66 \\
\hline Sérine $\ldots \ldots \ldots \ldots \ldots$ & 3,92 & 0,38 & 4,57 & 0,79 \\
\hline Acide glutamique $\ldots \ldots \ldots$ & 53,38 & 5,19 & 17,66 & 3,06 \\
\hline Proline $\ldots \ldots \ldots \ldots \ldots$ & 3,36 & 0,33 & 7,48 & 1,29 \\
\hline Glycine $\ldots \ldots \ldots \ldots \ldots$ & 4,31 & 0,42 & 4,17 & 0,72 \\
\hline Alanine ............. & 4,00 & 0,39 & 4,25 & 0,74 \\
\hline Valine ........... & 4,27 & 0,41 & 4,92 & 0,85 \\
\hline Isoleucine $\ldots \ldots \ldots \ldots \ldots$, & 3,38 & 0,33 & 3,81 & 0,66 \\
\hline Leucine $\ldots \ldots \ldots \ldots \ldots$ & 5,70 & 0,55 & 6,69 & 1,16 \\
\hline Tyrosine $\ldots \ldots \ldots \ldots$ & 3,06 & 0,30 & 3,05 & 0,53 \\
\hline Phenylalanine $\ldots \ldots \ldots$ & 3,88 & 0,38 & 4,36 & 0,75 \\
\hline Méthionine . . . . . . . & 2,35 & 0,23 & 1,85 & 0,32 \\
\hline Cystine $\ldots \ldots \ldots \ldots \ldots$ & 1,87 & 0,18 & 1,90 & 0,33 \\
\hline $\begin{array}{c}\text { Matières azotées } \\
\text { en p. } 100 \text { de l'échantillon }\end{array}$ & 9,72 & & 17,31 & \\
\hline
\end{tabular}

Il a été procédé, pour chaque critère étudié, à une analyse de variance permettant de déceler l'effet éventuel de l'apport de lysine supplémentaire.

En complément de cette étude, les performances de croissance d'un porcelet représentatif de chaque portée ont été notées jusqu'à un poids classique d'abattage ( Ioo kg environ). De plus, les performances de reproduction des truies ont été enregistrées à la seconde mise bas, les conditions d'alimentation et d'environnement ayant été, dans les deux cas, similaires pour tous les animaux après le sevrage de la première portée.

\section{RÉSULTATS}

\section{I. - Variations de poids et performances de reproduction des truies}

Dès le début de leur gestation, l'ensemble des truies des lots 2, 3 et 4 a une croissance apparente plus rapide que les truies vides du lot I (fig. I). Au moment de la mise bas, la différence de poids de $9 \mathrm{~kg}$, constatée entre les lots I et 2 , n'est pas signi- 
ficative, alors qu'est significatif l'écart de $\mathrm{I}_{4} \mathrm{~kg}$ enregistré en faveur des truies du lot 3, vis-à-ris de celles du lot $\mathrm{I}$, ces deux groupes d'animaux recevant le même régime et ne différant que par l'état physiologique. Ce phénomène est bien connu. Il est, en partie, expliqué par la prise de poids correspondant à la portée et aux annexes.

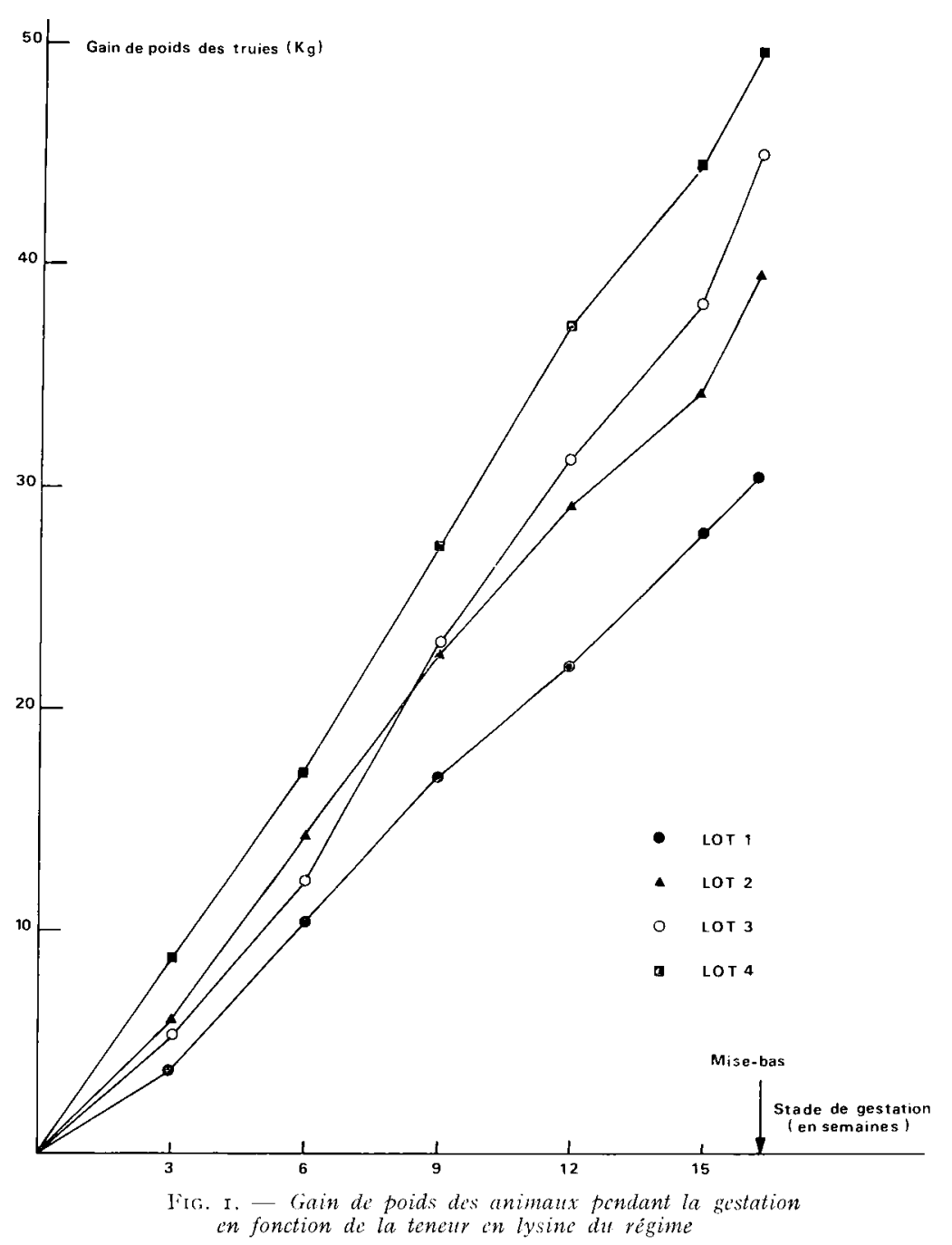

D'autre part, l'accroissement du taux de lysine dans le régime des truies gestantes a pour effet d'augmenter, parallèlement, le gain de poids net au cours de la gestation. Les différences ne sont pas significatives mais il est à remarquer que le gain net des truies gestantes des lots 2 et 3 a tendance à être plus faible que le gain de poids correspondant à la croissance des truies vides alors que celui du lot 4 lui est au contraire supérieur.

Le nombre de porcelets nés vivants par portée qui varie beaucoup dans chaque 
lot, ne paraît pas affecté par le régime de gestation, la différence observée n'étant pas significative. Par contre, il n'en est pas de même du poids moyen des porcelets à la naissance qui augmente de $27^{\circ}$ granmes quant on porte le taux de lysine de 0,23 à 0,42 p. Ioo dans le régime de base des truies gestante; (tableau 3). Cette anlélioration du poids moyen des porcelets est maintenue au taux le plus élevé (0,62 p. I0o) de lysine dans la ration (lot 4 ). Pas plus qu'à la mise bas le nombre de porcelets par portée à 3 semaines et au sevrage ( 5 semaines) n'est modifié par le régime de gestation (tableau 4). Par contre, le poids nnoyen à 35 jours des porcelets du lot 2 se révèle inférieur à celui des animaux de: lots 3 et + . Ia différence significative entre les lots 2 et 4 résulte d'une différence constante entre les gains de poids des porcelets des deux lots durant la lactation, associée à une dépression de leur consommation. Il est à signaler, toutefois, que les animaux du lot 3 ont une vitesse cle croissance plus faible que ceux du lot 4 , durant les trois première; semaines de vie, ce qui explique l'écart non significatif des poids au sevrage des animaux des deux lots considérés.

TABI,YAU 3

Tariations au poids des animanx au cours de la gestation 't performancis de reproduction à la mise bas

\begin{tabular}{|c|c|c|c|c|c|c|}
\hline Tencur en lysine (引u régine $\left(\begin{array}{c}0 \\
0\end{array}\right)$ & $\begin{array}{l}1,101 \\
(1, i=2\end{array}$ & $\begin{array}{c}\text { Lot }: 1 \\
11,23\end{array}$ & $\begin{array}{l}\text { Lot : } \\
0,1:\end{array}$ & $\begin{array}{l}\text { Lot it } \\
0, t i-2\end{array}$ & \multicolumn{2}{|c|}{$\begin{array}{l}\text { Signification } \\
\text { statistique } \\
s \vec{x}()(\mathbf{1})\end{array}$} \\
\hline & & & - & & & \\
\hline Poids des animaux a la saillio (kg) & 1117,7 & 105,6 & $111, r$ & $101 ;, 0$ & $1,\{$ & $(6, i j)$ \\
\hline Gain total en lli jours (kg).... & $: 31,-\pi$ & $39,7^{\prime \prime b}$ & $14,8 b c$ & $49,8 c$ & 3,5 & $(22,2,3)^{* * *}$ \\
\hline Gain net en 11 't jours $(k, g) \ldots$ & $: 31, \overline{7}$ & $2 ;, i$ & $2 x, 2$ & $: 3,7$ & $8,1)$ & $(27,1) \times S$ \\
\hline - & & - & -- & & & \\
\hline $\begin{array}{l}\text { Combre de porcelets nés vivants } \\
\text { par portée } \ldots \ldots \ldots \ldots \ldots \ldots \ldots\end{array}$ & & $R, '$ & $\therefore, 5$ & $7, \bar{i}$ & 0,6 & $(18,8) \mathrm{NS}$ \\
\hline $\begin{array}{l}\text { Poicls moven des porcelets it lat } \\
\text { naissance }(k g) \ldots \ldots \ldots \ldots \ldots\end{array}$ & & $0,98 a$ & $1,25,3^{2}$ & $1, \because 1^{\prime \prime}$ & 0,07 & $(15,54)^{*}$ \\
\hline $\begin{array}{l}\text { Poids total de la portée ì la mais- } \\
\text { sance }(\mathrm{kg}) \ldots \ldots \ldots \ldots \ldots\end{array}$ & ${ }^{-}$ & 8,11 & $10,-i i$ & $?, 1:$ & $0, \pi$ & $(\because 2, \geq 23) \mathrm{N}$ \\
\hline
\end{tabular}

(1) $s \bar{x}($ ) : écurt-type de la noyenute (coefficient de variation).

l.es valeurs suivies de la méme lettre ne diffèrent pas entre elles au seuil $\mathrm{P}<0,0.5$.

NS: differences non signitiratives.

* : différences significatives au scuil $\mathrm{P}<0,05$.

** : differences significatives au seuil $P<0,11$.

Ces différences entre les lots se retrouvent, dans une certaine mesure, quand on rassemble les données précédentes (nombre et poids des porcelets à la naissance ou au sevrage) dans le poids total de la portée aux deux stades envisagés. Mais, compte tenu de la variation importante intra-lot, de ces critères, les différences observées ne sont plus significatives.

Enfin, la consommation des mères durant la lactation semble plus importante dans le lot 2 , leur perte de poids, par contre, plus faible. 
TABI,EAU 4

Perfornances antechniques des truies it de leur porter au cours de la lactation

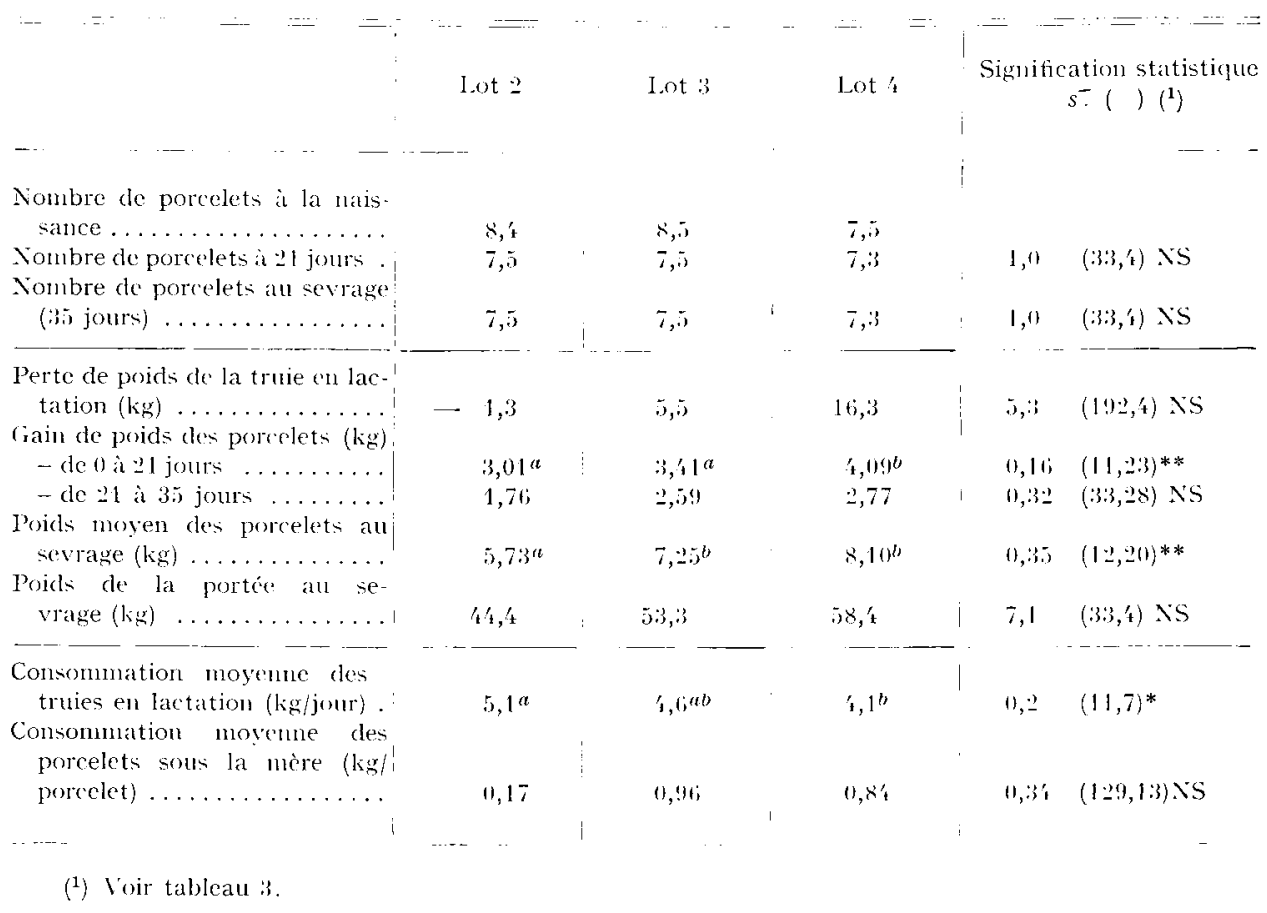

\section{2. - Ctilisation digestive des nutriments et rétention azotée}

Durant la période expérimentale en cages de digestibilité, les consommations journalières de matière sèche et de matières azotées ont été similaires dans les quatre lots soit, respectivement, I 785 et $2 \mathrm{I} 6 \mathrm{~g}$. La digestibilité apparente de la matière sèche ou des matières azotées ne semble pas modifiée ni par le régime de gestation, ni par l'état physiologique des animaux (tableau 5). Il apparaît pourtant une légère amélioration, non significative, de la digestibilité apparente des matières azotées avec le régime le plus riche en lysine.

Par contre, la quantité journalière d'azote retenu, à ce stade, est significativement plus élevée pour les truies du lot 4 , conséquence d'une excrétion azotée plus faible dans l'urine, en comparaison avec les truies des 3 autres lots (tableau 6).

Il s'ensuit que le coefficient de rétention de l'azote, qui traduit le pourcentage d'azote absorbé retenu par l'animal, est modifié dans le même sens.

\section{3. - Variations de's concentrations e'n acides aminés libres du sang}

Les variations des concentrations en acides aminés libres du sang suivant le le stade de gestation, l'état physiologique et la teneur en lysine du régime sont présentées dans le tableau 7. 


\section{TABLEAU 5}

Utilisation digestive de la matière sèche et de l'asote ingérés Stade de gestation correspondant à la période des collectes : 80 jours.

Nombre d'animaux par lot : 5

Matière sèche ingréré $(\mathrm{g} / \mathrm{j}) \ldots \ldots . \quad 17 \mathrm{ki}$

Matière sèche fécale $(\mathrm{g} / \mathrm{j}) \ldots \ldots$. CUI) $\left({ }^{2}\right)$ de la matière sèche $(\%)$.

Azote ingéré (gN/jour) .......

Azote fécal $(\mathrm{g} / \mathrm{jour}) \ldots \ldots \ldots \ldots$

CII $\left({ }^{2}\right)$ azote $\left(\begin{array}{l}0 \\ 0\end{array}\right) \ldots \ldots \ldots \ldots$
I.st 1

$$
200
$$

$\times 5,4$

$3 / 4$

6,4

si,

$\operatorname{Lot} 2 \mid \operatorname{Lot} 3 \quad$ Lot't

Signification statistique $s \bar{x}(1)$

(1) $s \bar{x}($ ) : 'cart-type de la moyemne (coefficient de variation)

NS : différences entre les lots non significatives.

(2) $\mathrm{C}(\mathrm{J})$ : coefficient d'utilisation digestive apparent.

\section{TABLFAU 6}

L'tilisation métabolique de l'azotc absorbé

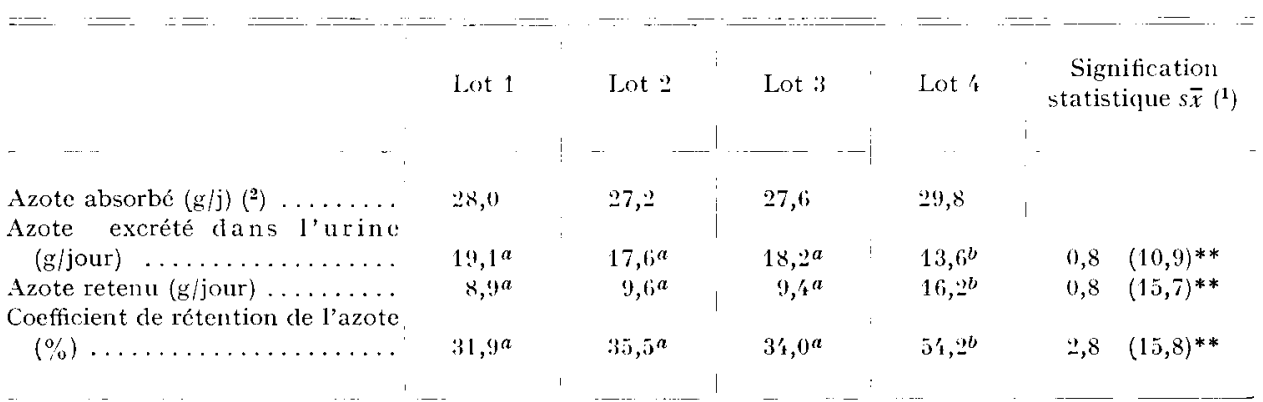

(l) $s \bar{x}($ ) : écart-type de la moyenne (coefficient de variation).

Les valeurs suivies de la même lettre ne diffèrent pas entre elles au seuil $P<0,05$

NS: différences non significatives

** : différences significatives au seuil $\mathbf{P}<0,01$.

$\left.{ }^{2}\right)$ : d'après les valeurs du tableau précédent. 


\section{TABI,EAU 7}

Variations des concentrations en acides aminés libres du sang 'n fonction du stade de gestation et de la teneur cn lysinc du régime

(en $\mathrm{mg} / \mathrm{l}$ oo $\mathrm{g}$ de sang frais)

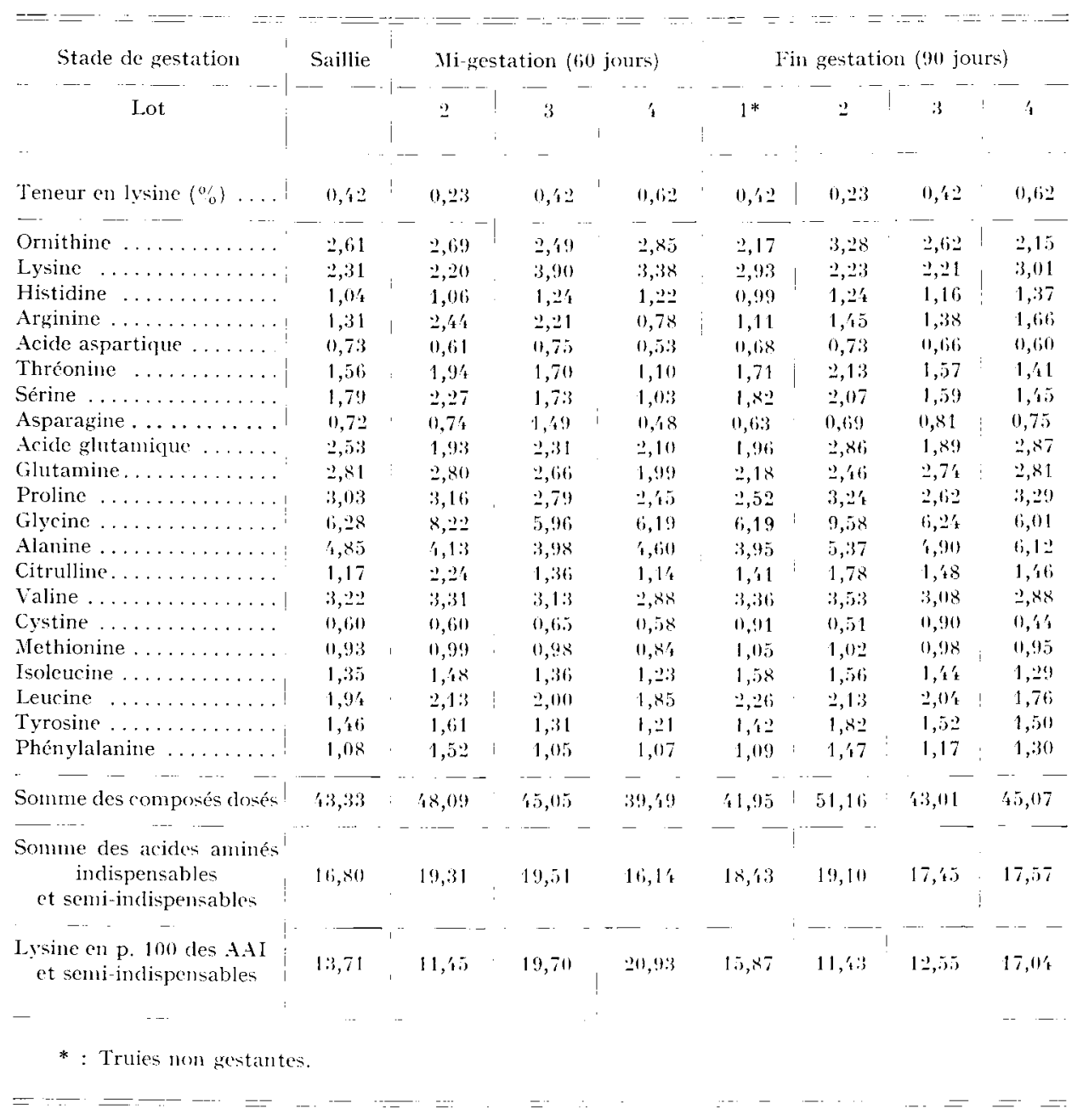

En ce qui concerne les truies gestantes (lots 2, 3, 4), l'augmentation du taux de lysine dans le régime provoque, vers le milieu de la gestation, une élévation de la concentration de lysine libre du sang et une diminution de certains acides aminés indispensables (thréonine, valine, isoleucine, leucine, acides aminés aromatiques). Par contre, à la fin de la gestation, l'augmentation de la concentration de lysine libre du sang n'est effective qu'avec le taux le plus élevé de lysine dans le régime. A ce stade, les concentrations de l'ensemble des acides aminés indispensables diminuent avec le taux intermédiaire de supplémentation et cette diminution est maintenue au taux le plus élevé. 
L'augmentation du taux de lysine dans le régime a pour effet, également, de faire baisser les concentrations en certains acides aminés non indispensables, comme la sérine et surtout la glycine, aux deux stades de l'étude. Fn ce qui concerne les truies vides (lot I), la comparaison des concentrations en acides aminés libres du sang à deux stades différents de leur croissance, espacés de 3 mois, fait ressortir une accumulation en certains acides aminés indispensables libres dans le sang (lysine, thréonine, cystine, isoleucine, leucine). Au contraire, certains acides aminés indispensables, (histidine, arginine) ou non indispensables (acide glutamique, proline, alanine) présentent une concentration dans le sang plus faible quand l'animal est plus âgé.

Enfin, la confrontation de l'aminoacidémie libre des animaux gestants ou non, recevant le même régime, montre qu'il n'existe que peu de différences dues à l'état physiologique. L'état de gravidité, chez la Truie, a surtout, pour conséquence, une diminution des concentrations en certains acides aminés indispensables libres du sang (1ysine, thréonine, valine).

\section{4. -- Composition en matières azotées et en acides aminés du colostrum}

Les échantillons de colostrum ont été analysés après avoir été groupés par lot ; les résultats de composition en matières azotées et en acides aminés (sauf les acides aminés soufrés) figurent dans le tableau 8.

TABI,EAU 8

Composition en acides aminés du colostrum suivant la teneur en lysine du régime de gestation

(en g p. $16 \mathrm{gN}$ )

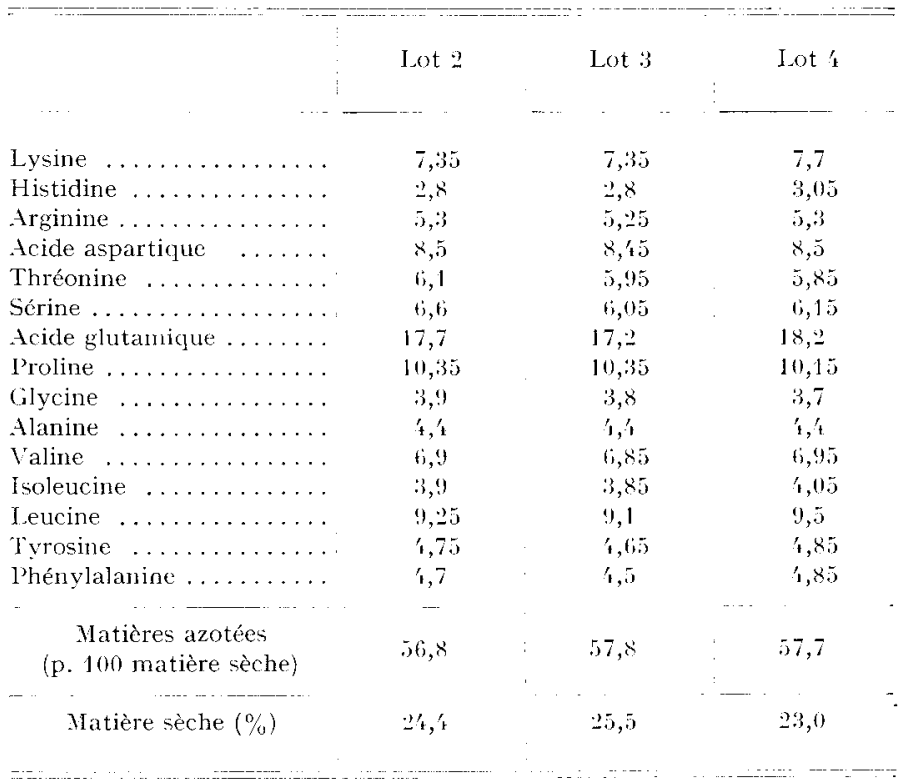


Les teneurs du colostrum en matières azotées sont comparables entre les lots. Il apparaît, cependant, une légère augmentation de la somme des acides aminés indispensables et semi-indispensables dans le colostrum des truies du lot 4, par rapport aux deux autres lots. Cet écart porte, principalement, sur les teneurs en 1ysine et leucine.

\section{5. - Croissance post-serrage des porcelets}

L'effectif de porcelets mâles castrés, dont la croissance postérieure au sevrage a été enregistrée, est représentatif de l'ensemble des animaux de chaque lot si l'on se fonde sur leur poids moyen à la naissance et au sevrage. Ces animaux ont été élevés dans les mêmes conditions, de logement et d'alimentation jusqu'à l'abattage fixé à un poids voisin de roo $\mathrm{kg}$. Les données recueillies dans le tableau 9 montrent qu'à ce poids d'abattage, l'âge des animaux est d'autant plus élevé que le poids moyen au sevrage est plus faible.

\section{TABLEAT 9}

Effet de la teneur en lysine du régime maternel de gestation sur los performances de croissance de la portée

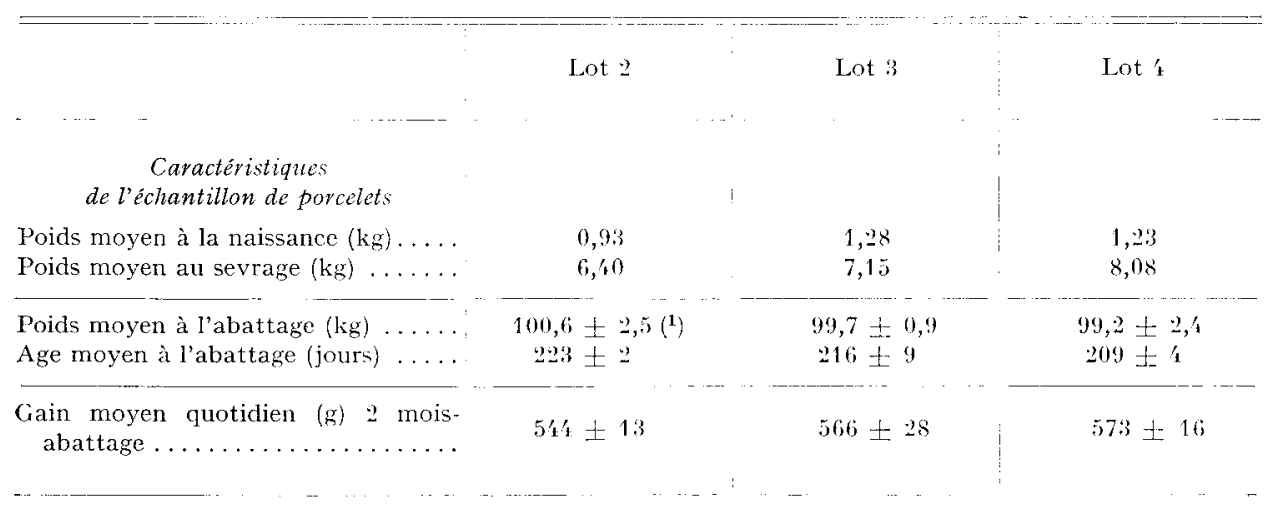

(1) Valeur moyenne suivie de l'écart-type de la moyenne.

Quoique légèrement inférieur dans le lot 2, le gain moyen quotidien des animaux, entre l'âge de 2 mois et le stade d'abattage ne semble pourtant pas modifié par leur poids initial.

\section{DISCUSSION E'T CONCLUSIONS}

Les recommandations formulées, quant au besoin en acides aminés de la Truie gestante (N. R.C., I973) sont fondées, en grande partie, sur les expériences de RIPPEL et al., (I $965 a$ et $b$ ). Ces auteurs associent le besoin en acides aminés indispensables à une rétention maximale d'azote corporel en fin de gestation. Le problème 
se pose de savoir si, parallèlement à l'augmentation de la rétention azotée maternelle, les performances de reproduction à court terme (nombre et poids des porcelets à la mise bas) ou à moyen terme (croissance des porcelets sous la mère, performances ultérieures de reproduction) sont accrues. Or, les travaux de PIKE (I970) démontrent qu'un supplément d'apport azoté, donc un niveau de rétention azotée plus élevé n'est bénéfique qu'à la mère sans incidence sur la portée. De plus, les observations de Poxp et al., (I958 $a$ et $b$ ), réalisées cependant sur un faible effectif d'animaux, soulignent le peu d'influence, sur les performances de reproduction, d'un régime protéiprive pendant toute ou partie de la gestation. Dans cette optique, il est possible d'envisager une réduction de l'apport azoté qualitatif en gestation et le but de la présente expérience a été de déterminer les effets éventuels d'une carence partielle en lysine dans le régime de truies nullipares.

\section{I. - Effets à court terme d'une réduction de l'apport de lysine en gestation}

La présence, dans le dispositif expérimental, d'un lot de truies non gestantes (lot I), recevant le même régime que les truies gravides du lot 3 , met en évidence l'absence d'anabolisme gravidique défini comme la différence de gain de poids entre truies gestantes et non gestantes qui n'est pas seulement expliquée par le poids des produits de la conception. Ce phénomène, qui était connu chez d'autres espèces, a été démontré, chez la Truie adulte, par SALMON-LEGAGNEUR et JAcQUOT (I96I), LODGE et al., (I96I), Rombauts (Ig62). Il apparaît même avec des régimes alimentaires qui ne permettent pas à des truies non gestantes de modifier leur poids (SALMON-LEGAGNEUR et RÉRAT, I962). L'anabolisme de gestation se trađuit, en particulier par une amélioration de la rétention azotée, surtout peu de temps avant la mise bas (ELSLEY et al., I 966). Ce n'est pas le cas, dans cette étude, si l'on compare les performances des lots I et 3. Dans des conditions de rationnement énergétique ou azoté légèrement plus faibles que celles préconisées par le N. R. C. (I973), on peut se demander si ce phénomène d'anabolisme gravidique existe chez la Trtuie nullipare, saillie à un poids voisin de $100 \mathrm{~kg}$, et dont la croissance n'est pas terminée. Ceci rejoint, en partie, les conclusions d'autres travaux (DE WILDE, VAN SPALNDONCK, VANSchoubroek, I973; ETIENNE et HENRY, I973).

L'accroissement du taux de 1ysine dans la ration a pour premier effet d'augmenter, linéairement, le gain total de gestation, ce qui n'avait pas été trouvé par SALMON-IEGAGNEUR et DUÉE (1972) sur truies adultes. L'augmentation du gain net est, par contre, moins marquée entre le lot 2 et le lot 3. Mais, dans le même temps, le poids moyen des porcelets augmente significativement, sans que leur nombre ne soit modifié. Ceci démontre, d'une part, que la croissance des porcelets in utero est ralentie quand le régime de gestation est partiellement carencé en lysine et, d'autre part, qu'un supplément en lysine est bénéfique d'abord à la portée et ensuite à la croissance maternelle. L'effet néfaste sur les performances de reproduction d'une carence azotée quantitative ou qualitative n'a jamais été clairement démontré. Si le nombre de porcelets ne semble pas être affecté par la teneur en matières azotées de la ration, leur poids moyen à la naissance peut, dans des conditions extrêmes de rationnement, être diminué (Pons et al., I968). Toutefois, ces résultats ne sont pas confirmés par les travaux de Rippei, et al. (I965), NIELSEN (I970) ou de GEETER et al. (1972). Dans le cas d'une carence en lysine du régime maternel de gestation, 
1a diminution du poids moyen des nouveau-nés est en accord avec les résultats chez d'autres espèces, la ratte en particulier (NIryama ct al., I973). Il faut souligner, de plus, que les performances des truies lors de leur seconde gestation oi elles recevaient un régime standard ne sont pas différentes suivant le régine de leur prenière gestation et sont, en moyenne, pour le gain net de gestation de $33,3 \mathrm{~kg}$, pour le nombre de porcelet i virants à la naissance de 9,3 , et pour le poids moyen des porcelets à la naissance de $I, 3.5 \mathrm{~kg}$.

Cette augmentation du poids moyen des porcelets à la naisiance avec le taux intermédiaire de supplémentation en lysine est maintenue avec le taux le plus élevé et, clans ce cas, l'augmentation dı gain net des mères est plus accentuée. Parallèlement, l'essai de détermination de la rétention azotée en fin de gestation confirme, à un instant donné, les différences entre les lots observées précédemment. In effet, on ne note pas d'amélioration de la rétention azotée entre les lots 2 et 3 ce qui souligne le peu de différence dans les croissances propres des mères des deux lots, compte tenu du fait que les produit; de la conception (portée, placenta et liquides), riches en eau, n'interviennent que très faiblement dans le bilan azoté final. Par contre, il existe une bonne concordance entre la rétention azotée des truies du lot + et leur gain propre de poids au cours de la gestation. In se fonclant uniquement sur les résultats de nise bas, il s'avère, donc, qu'il n'est pas nécesiaire d'obtenir une rétention azotée maximale en fin de gestation pour asurer l'optimum des performances de reproduction.

inn relation avec les clifférents critères précéclents, l'examen des variations des concentrations en acide; aninés libres du sang peut représenter un moyen d'étude du besoin en un acide aniné essentiel, dans la mesure où les prélèvements de sang sont effectués à un moment oì la concentration en chacpue acide aminé dans le sang représente l'équilibre entre l'apport alimentaire et la soutstraction du pla'ma qui est fonction du besoin de l'organisme en cet acide anniné (RÉRAT, I97I, I972). On peut penier que ces conditions sont réalisées dans le cas présent (prélèvements effectués 6 h 30 après le repas), si l'on se fonde sur les expériencess réalivées sur des animaux

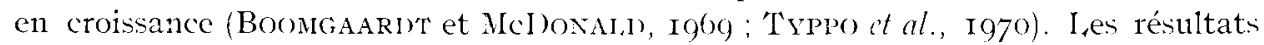
montrent que le; variation; de concentration du facteur limitant, la lysine, sont différentes aux deux stades étudiés. Il semble exister une évolution dans le besoin an cours de la gestation : l'accumulation de lysine libre dans le sang est visible dès le taux de 0,42 p. Ios, à 60 jours de gestation, mais ne peut être mis en évidence cu'au taux de 0,62 p. Ion après go jours de gestationl. Ces phénomènes concernant la lysine sont particulièrement nets quand on rapporte cet acide aniné à la somme des acicles aminés indispensables (tabl. 7). La diminution de concentration de l'ensemble des acide:s aninés indispensables, et plus particulièrement de certains d'entre enx comme la thréonine, semble indiquer une meilleure utilisation des protéines alinentaires lorsque le taux de lysine dans le régine augmente, ce qui est en accord avec des observations réalisées sur le Porc en croissance (PION at al., I97 I). Finfin, le besoin de fin de croissance petut être cléduit de l'évolution des concentrations en acides aninés libres chez les animaux non gestants. Ene accunulation plus importante des acides aninés indispensable; (la lysine et la thréonine, en particulier) est constatée au stade le plus tardif de la croissance, ce qui pourrait signifier un besoin de croissance plus faible.

I,es données sur les teneurs en matières azotées et en acides aminés du colostrum sont en accord avec la composition rapportée par I) Ú́. et Jravi (I973). L'absence 
d'effets marquants de la teneur en lysine du régime sur la composition du colostrum confirme les observations précédentes d'EiLIOTT et $a l$., (I97I).

En définitive, à travers les conclusions que l'on a pu tirer, il apparaît une bonne convergence entre les différents critères étudiés, ce qui est représenté par les réponses des principaux paramètres à la supplémentation en lysine de gestation (fig. 2).
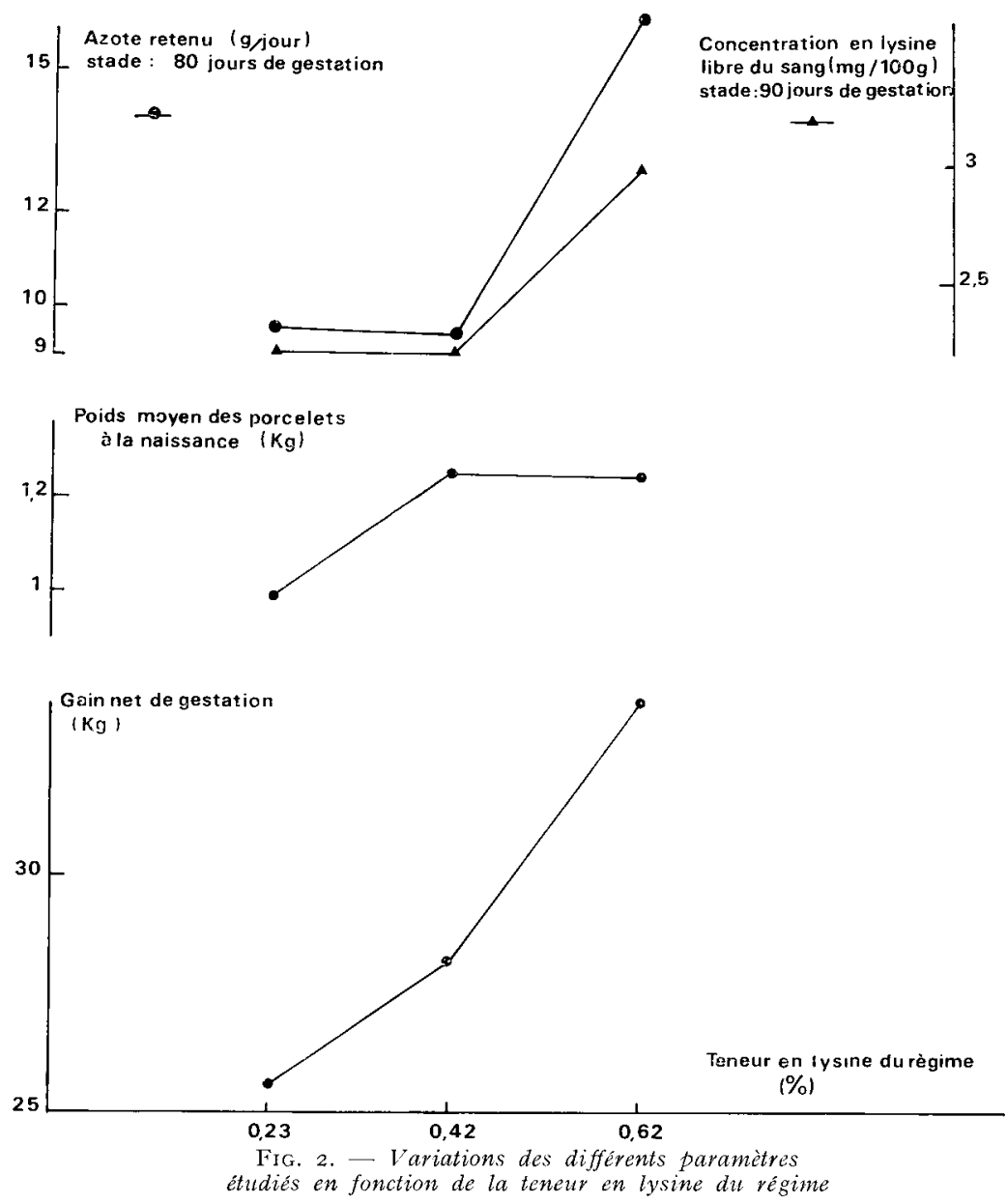

2. - Effets à moyen terme d'une réduction de l'apport de lysine*en gestation

Les travaux de SALMON-LEGAGNEUR (I965) ont démontré qu'il existait des relations nutritionnelles entre la gestation et la lactation. Dans le cas présent, les variations pondérales des truies au cours de la lactation sont en liaison avec le gain de poids durant la gestation. Plus le gain de poids est important en gestation, plus la perte de poids de lactation est élevée, à l'inverse de la consommation spontanée d'aliment par les animaux durant cette période confirmant en cela les résultats de SALMON-LEGAGNEUR (i965).

Les différences de poids observées, à la mise bas, entre les porcelets du lot 2 , 
d'une part, et des lots 3 et 4 d'autre part, se retrouvent au moment du sevrage, avec des écarts plus accentués, sans que le nombre de porcelets, à ce stade, soit différent suivant les traitements. Il apparait, toutefois, que le poids moyen au sevrage des porcelets du lot 3 est plus faible que celui des porcelets du lot 4 . Ceci est la conséquence du ralentissement de leur croissance durant les trois premières semaines de vie, que l'on peut associer à la perte de poids des mères plus faible, donc à une diminution de leur production laitière. Ainsi, comme le souligne PoND (I973), le niveau d'apport azoté en gestation influence indirectement la production laitière de la mère, surtout pendant les premiers jours après la mise bas. Cette diminution de la production laitière a pour causes soit la dépression des réserves corporelles de gestation qui interviennent, en particulier pour les nutriments azotés, dans la synthèse des constituants du lait au début de la lactation (SALMON-LEGAGNEUR, I965), soit le retard dans le développement de la glande mammaire qui a lieu en fin de gestation à un moment où le besoin azoté quantitatif et qualitatif est probablement plus élevé.

Après 3 semaines de lactation, la croissance des porcelets qui reçoivent un aliment de démarrage n'est plus différente dans les lots 3 et 4 , mais reste légèrement inférieure dans le lot 2 , du fait même d'une consommation plus faible des jeunes. A cet âge d'ailleurs, l'influence de la consommation d'aliment, par rapport à l'ingestion de lait, sur la croissance des animaux est prépondérante (Aumaitre, SALMoNLEGAGNEUR, I96I).

Enfin, les performances de croissance des animaux, après le sevrage ne sont pas différentes suivant la teneur en lysine du régime maternel de gestation, ce qui est en accord avec les précédentes observations de Livingstone et al., (I966), Pond et al., (I968 b), de GEETER et al. (r973). Il subsiste, néanmoins, un écart dans l'âge des animaux à l'abattage, conséquence surtout des différences observées aux premiers stades de la croissance.

En conclusion, dans la détermination du besoin en acides aminés de la truie gestante, la présente étude a souligné l'intérêt de considérer les performances de reproduction dans leur ensemble, en relation avec d'autres critères comme les variations de l'aminoacidémie libre. Il sera nécessaire de poursuivre cette expérience avec des doses plus rapprochées de lysine afin de mieux préciser le besoin chez la Truie nullipare qui, compte tenu des résultats, se situe entre 0,42 et 0,62 p. Ioo du régime.

Reçu pour publication en février 1975.

\author{
SUMMARY
}

\title{
LYSINE REQUIREMENT OF NULLIPAROUS GILTS
}

The aim of the present study was to determine the lysine requirement of nulliparous pregnant gilts. For that purpose, 4 groups comprising 6-7 Large-White gilts and including a control group composed of empty sows were compared during one reproductive cycle. The comparison was based on the weight variations of the animals, the reproductive performances relative to digestibility and nitrogen retention in late pregnancy (80 days) and the changes in the blood level of free amino acids. The basal diet used during pregnancy contained $3360 \mathrm{kcal}$ of digestible energy per $\mathrm{kg}$ dry matter, $10.5 \mathrm{p}$. I 00 crude protein and $0.23 \mathrm{p}$. I 00 of lysine and was offered at a constant level of $2 \mathrm{~kg}$ per day. It was supplemented with various levels of industrial L-lysine $(0,0.2,0.4$ 
p. Ioo of the diet) corresponding to the thrce groups of pregnant gilts (groups $2,3,4$ ). The empty gilts of group I received the same diet as these of group 3. During lactation, all animals received ad libitum a ration containing $16 \mathrm{p}$. Ioo protein.

The addition of lysine to the basal diet during pregnancy brought about an increase in the weight gain of the animals during this period (gestation gain (kg): group $2: 39,7$, group $3: 44.8$, group $4: 49.8$. In comparison with the lysine deficient group, a supplementation with this amino acid (0.2 p. Ioo) led to an increase in the mean weight of the piglets at birth (group $2: 0.98 \mathrm{~kg}$, group $3: 1.25 \mathrm{~kg}$ ) without affecting the number (group $2: 8.4$, group $3: 8.5$ ). However, the real growth of the gilts was lower than that of the controls (net gestation gain $(\mathrm{kg}):$ group $I: 30.7$, group $2: 25.6$, group $3: 28.2)$.

A larger lysine supplementation (o.t p. Ioo of the diet) gave a maternal growth similar to that of the controls (net gain of the group $+: 33.7 \mathrm{~kg}$ ), without improving the reproductive performances at farrowing (litter size : 7.5 mean weight of the piglets : $1.24 \mathrm{~kg}$ ). This resulted in a higher nitrogen retention in late pregnancy $(16.2 \mathrm{~g}$ of nitrogen per day in group + versus 8.9, 9.6, 9.4 , respectively in groups $\mathrm{I}, 2,3$ ).

The unfavourable effect of lysine deficiency during pregnancy also affected the weight of the piglets at weaning (number and mean weight $\langle\mathrm{kg}$ ) : group $2: 7 \cdot 5,5 \cdot 3 ;$ group $3: 7 \cdot 5,7 \cdot 25 ;$ group 4 : $7 \cdot 3,8.10)$.

Study of the variations in the blood levels of free amino acids showed that the lysine requirement was higher in late pregnancy.

The growth performances of the piglets after weaning were not affected by the lysine content of the maternal diet during pregnancy, but a delay in growth subsisted in the deficient group at slaughtering ( $100 \mathrm{~kg}$ ).

The results of this study show that the level of lysine must not be too much reduced in the diet of the gilt during pregnancy.

\section{RÉFÉRENCES BIBLIOGRAPHIQUES}

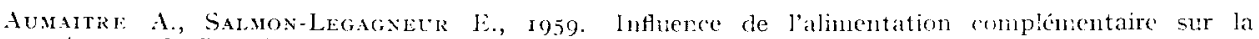
croissance du Porcelet avant le sevrige. Ann. Zontech., 10, I27-1 40.

Benson J. V., Gordon M. J., Pattreson J. A., 1967. Accelerated chromatographic analysis of amino acids in physiological fluids containing glutanine and asparagine. Anal. Biochem., 18, 228-240.

Boomgarot J., Mclonald 13. E., I969. Comparison of fasting plasma aminoacid patterns in the pirs, rat and chicken. Can. J. I'hysiol. I'harmacol, 47, 392-395.

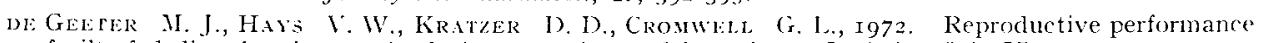
of gilts fed diets low in protein during gestation and lactation. J. Anim. Sci., 35, 772-777.

de Geeter M. J., Hass V. W., Kratzer 1). D., Cromwell G. I, , 973 . Jostweaning performance of pigs gilts from fed diets high and low in protein during gestation and lactation. J. Anim. Sci., 3y, 459-462.

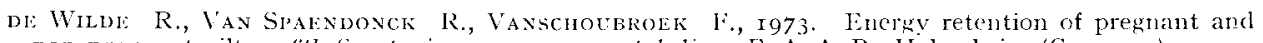
non pregnant gilts. tith Symposimm on energy motabolism, E. A. A. P., Hohenheim (Germany).

Dúé P. H., jt:NG J., I973. Composition cul acides aminés du lait de truir. Ann. Zootech., 22, $243-247$.

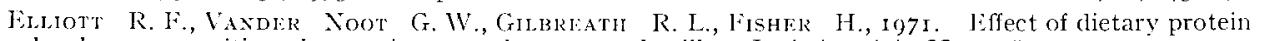
level on composition changes in sow colostrum and milk. J. Anim. Sici, 32, i 28 - I 37.

Filsiey 1:. W. H., Anderson I). M., McDonal.d J., McPhlirson R. M., Smart R., ig66. A comparison of the live-weight changes, nitrogen retention and carcass composition of pregnant and non presnant gilts. Anim. Prod., 8, 39I-400.

Jitenni; M., Hunry Y, I973. Influence de l'apport énergétique sur l'utilisation digestive et métabolique des nutriments et les performances de reproduction chez la Truic gestante nullipare. Ann. Zootech., 22, 3 I I-326.

I.ivingstone R. M., Mclihekson R. M., Jisher li. W. M., Licas I. A. M., Lodge G. A., ig66. note on the effect of protein concentration in the diets of pregnant sows on the performance and carcass quality of their progeny. Anim. Prod., 8, 337-339.

Lovge (x. A., Mg,Donald I., McPherson R. M., ig6r. Weight changes in sows during pregnancy and lactation. Anim. Prod., 3, 260-275.

MoustgaArd J., I959. Nutrition and reproduction in domestic animals in Roproduction in donnestic animals, vol. 2 , ed. by Cole and Cupps. Acad. Press, New York, I 70-223.

Nielsex H. I:, I970. Recent Danish Investigations on the nutrition of sows. Proc. of RITENA IIallorea, I 01 - I 16 . 
Nityama Y., Kishi K., Endo S., Inove G., I973. Effect of diets devoid of one essential amino acid on pregnancy in rats maintained by ovarian steroids. J. Nutr., 103, 207-2I2.

N. R. C., 1973. Nutrient requirements of swine. National Academy of Sciences, Washington.

PAWLAK M., Pion R., I 968 . Influence de la supplémentation des protéines de blé par des doses croissantes de lysine sur la teneur en acides aminés libres du sang et du muscle du Rat en croissance. Ann. Biol. anim. Bioch. Biophys., 8, 517-530.

PIKE 1. H., 1970. The effect on nitrogen utilization in the sow of variation in dietary protein concentration and pattern of feeding in pregnancy. J. Agric. Sci., 74, 209-215

Pion R., liauconneau G., 1966. Les acides aminćs des protéines alimentaires. Méthodes de dosage et résultats obtenus. Cahier A.E. C. n ${ }^{\circ} 6$, I 57 -I 75 .

Pion R., Prugnaud J., Henry Y., Rérat A., ig7i. Influence de la teneur en thróonine du réginie sur l'aminoacidémie libre du Porc en croissance. 220 Réunion Annuelle F. E. Z., Versailles (France).

Pond W. G., 1973. Influence of maternal protein and energy nutrition during gestation on progeny performance in swine. J. Anim. Sci., 36, I75-182.

Pond W. G., Wagner W. C., Dunn J. A., Walker I. F., ig68 a. Reproduction and early postmatal growth of progeny in swine fed a protein-free diet during gestation. J. Nutr., 94, 309-3r6.

Pond W. G., Dunn J. A., Wellington G. H., Stouffer J. R., Van Vleck L. D., ig68 b. Weight gain and carcass measurements of pigs from gilts fed adequate vs. protein-free diets during gestation. J. Anim. Sci., 27, 1583 -1586.

fond W. G., Strachan D. N., Sinha Y. N., Walkfr E. F., Dund J. A., Barnes R. H., I969. Effect of protein deprivation of swine during all or part of gestation on bit th weight, postnatal growth rate and nucleic acid content of brain and muscle of progeny. $J, N u t r, 99,6 \mathrm{I}-67$.

Rérat A., r97I. La valeur biologique des protéines: quelques acquisitions récentes. Ann. Zootech., 20, $193-246$.

RÉrat A., 1972. Protein nutrition and metabolism in the growing pig. Nutr. Abstr. Rez., 42, I3-39.

Rrppel R. H., ig67. Protein and amino acid nutrition of gravid swine. J. Anim. Sci, 26, 526-532.

Rippel R. H., Harmon B. G., Jensen A. H., Norton H. W., Becklir D. E., I965a. Essential amino acid supplementation of intact proteins fed to the gravid gilt. J. Anim. Sci., 24, 373-377.

Rippel R. H., harmon B. G., Jensen A. H., Norton H. W., Becher D. F., i965 b. Some amino acid requirements of the gravid gilt fed a purified diet. J. Anim. Sci., 24, 378-382.

Rombauts P., r962. Évolution de l'anabolisme gravidique chez la Truie en fonction de l'âge de l'animal Ann. Zootech., 11, 39-5I.

Salmon-Legagneur E., Jaçuot R., I96r. Modifications corporelles entrainées par l'anabolisme gravidique chez la Truie. C. R. Acad. Sci, Paris, 544-545.

Salmon-Legagneur E., I965. Quelques aspects des relations nutritionnelles entre la gestation et la lactation chez la Truie. Ann. Zootech., 14, no H. S., I-I 37.

Salmon-Legagneur F., Duće P. H., ig72. Essai de supplémentation en lysine d'un régime à base de céréales chez la Truie en gestation et en lactation. Journées Rech. Porcine en France, Paris, ed. by I. T. P., I57-I6I.

Salmon-Legagneur E., Rérat A., ig62. Nutrition of the sow during pregnancy. In Nutrition of pigs and poulty, Butterworths, Morgan and Lewis eds., London, 206-223.

Spackman D. H., Stein W. H., Moore S., i958. Automatic recording apparatus for use in the chromatography of amino acids. Anal. Chem., 30, I190-I206.

Typpo J. T., Meade R. J., Nordstrom J. W., Stockland W. L., I97o. Influence of time of fast on concentrations of free amino acids in plasma and total amino acids in liver protein of young swine. J. Anim. Sci., 31, $885^{-893 .}$ 